Article

\title{
Local Limit Theorem for the Multiple Power Series Distributions
}

\author{
Arsen L. Yakymiv
}

Steklov Mathematical Institute of Russian Academy of Sciences, 8 Gubkina St., Moscow 119991, Russia; arsen@mi-ras.ru

Received: 30 September 2020; Accepted: 13 November 2020; Published: 19 November 2020

\begin{abstract}
We study the behavior of multiple power series distributions at the boundary points of their existence. In previous papers, the necessary and sufficient conditions for the integral limit theorem were obtained. Here, the necessary and sufficient conditions for the corresponding local limit theorem are established. This article is dedicated to the memory of my teacher, professor V.M. Zolotarev.
\end{abstract}

Keywords: multiple power series distribution; integral limit theorem; local limit theorem; Tauberian lemma; $R$-weakly one-sided oscillation of the multiple sequence at infinity along the given multiple sequence

\section{Introduction}

Let $(a(i) \geq 0, i=0,1,2, \ldots)$ be a sequence with

$$
B(x)=\sum_{i=0}^{\infty} a(i) x^{i}<\infty
$$

for $x \in(0,1)$. The trivial case $a(i) \equiv 0, i=0,1,2, \ldots$ is excluded. It is said that a random variable $\xi_{x}$ has a power series distribution iff

$$
\mathrm{P}\left\{\xi_{x}=i\right\}=\frac{a(i) x^{i}}{B(x)}
$$

for some $B(x)$ and for any $i \in Z_{+}$.

Power series distributions were introduced in the fundamental paper of Noack [1] (1950).

Systematic studies of their properties (moments, generating functions, convolutions, limit properties, statistical applications, etc.) began immediately. References may be found in the encyclopedias of Johnson, Kotz, and Kemp [2] (for the one-dimensional case) and Johnson, Kotz, and Balakrishnan [3] (for the multidimensional case). For example, the binomial, Poisson, negative binomial, and logarithmic distributions, as well as their multidimensional analogues are among the important distributions in this class.

Note that power series distributions are widely useful in a generalized allocation scheme (in the one-dimensional case). This scheme was introduced by V. Kolchin [4]. His results and, in particular, those obtained with the use of this scheme, play an important role in probabilistic combinatorics (see, for example, his books $[5,6])$. So, one can express distributions of various characteristics of random permutations $(\mathrm{a}(\mathrm{i})=1 / \mathrm{i})$, random mappings $\left.\left(\left(a(i)=i^{-1} \sum_{k=0}^{i-1} i^{k} / k !\right)\right)[5]\right)$, and random mappings with various constraints (on cycle length, height, component sizes, etc.; see, for example, the books of Timashev [7,8]), random trees, and random forests (i.e., random mappings with cycles of only unit length (see the book of Yu. Pavlov [9])) in terms of power series distributions. An analogue of Kolchin's generalized allocation scheme [4] with a bounded number of particles was introduced in the work of A.N. Chuprunov and I. Fezekash [10]. A corresponding multivariate scheme was 
recently introduced by A.N. Chuprunov, G. Alsaied, and M. Alkhuzani [11]. For another investigation of A.N. Chuprunov and his students, see the paper [11] and the references therein. We also note the successful work of the representatives of the Karelian Scientific Center in the study of the asymptotic properties of configuration graphs under the leadership and participation of Yu.L. Pavlov by I.A. Cheplyukova, M.M. Leri, and E.V. Khvorostyanskaya [12-17].

Suppose that $B(x)$ regularly varies as $x \uparrow 1$ with index $\varrho>0[18,19]$. It is known, in this case, that

$$
\mathrm{P}\left\{\xi_{x}(1-x) \leq y\right\} \rightarrow \int_{0}^{y} e^{-u} u^{--1} d u, \forall y \geq 0
$$

as $x \uparrow 1$. In addition, the corresponding local limit theorem is true when $a(i)$ is regularly varying at infinity with index $\varrho-1>-1$. See, for instance, Timashev [8].

The multidimensional integral limit theorem was obtained in [20]. It is supposed in [20] that the corresponding multiple power series regularly varies at the boundary point of its convergence (see Definition 2). In [21], it was shown that this condition is necessary and sufficient.

In this paper, we prove the corresponding local limit theorem. For this aim, we introduce in Section 2 some generalizations of multivariate regularly varying sequences in the orthant. Namely, the notion of R-weakly one-sided oscillatory sequences at infinity along some sequence (see Definition 3). This concept allows us to give adequate conditions for the validity of both the local limit theorem and the corresponding statement of Tauberian type (Lemma 2). The definition of multiple power series distribution and the main result are given in the next section (see Definition 1 and Theorem 1, respectively). Here, we also formulate the corresponding integral limit result from [21] as Lemma 1 . The statement of this lemma also gives the necessary and sufficient conditions but describes them in terms of regular variation of the power series $B(x)$ at the boundary point of their existence. Proofs of Lemma 2 and the main result (Theorem 1) are given in the Sections 3 and 4, respectively. In Section 5, we describe some previous results in this direction.

\section{Main Result}

\subsection{Some Notations}

We introduce the following notations. Let the vectors $x=\left(x_{1}, \ldots, x_{n}\right)$ and $y=\left(y_{1}, \ldots, y_{n}\right)$ belong to $R^{n}$. Denote $x y=\left(x_{1} y_{1}, \ldots, x_{n} y_{n}\right)$ and $x / y=\left(x_{1} / y_{1}, \ldots, x_{n} / y_{n}\right)$ (the last in the case, when $\left.y_{k} \neq 0 \forall k=1, \ldots, n\right)$. Put $\exp (x)=\left(\exp \left(x_{1}\right), \ldots, \exp \left(x_{n}\right)\right), \ln x=\left(\ln x_{1}, \ldots, \ln x_{n}\right)$. The notation $x \uparrow \mathbf{1}$ means that $x \rightarrow \mathbf{1}, x \in(0,1)^{n}$. Here $\mathbf{1}=(1, \ldots, 1)$. Set $R_{+}^{n}=\left\{x: x=\left(x_{1}, \ldots, x_{n}\right) \in\right.$ $\left.R^{n}, x_{k} \geq 0 \forall k=1, \ldots, n\right\}, Z_{+}^{n}=\left\{x: x=\left(x_{1}, \ldots, x_{n}\right) \in R_{+}^{n}, x_{k} \in Z_{+}=N \cup 0 \forall k=1, \ldots, n\right\}$. For $\alpha=\left(\alpha_{1}, \ldots, \alpha_{n}\right) \in R_{+}^{n}, x=\left(x_{1}, \ldots, x_{n}\right) \in R_{+}^{n}$ we use an abbreviation

$$
x^{\alpha}=\prod_{k=1}^{n} x_{k}^{\alpha_{k}}
$$

assuming that $0^{0}=1$. Let $\left(\eta_{k}, k \in N\right)$ be a sequence of random vectors (r.v.) from $R^{n}$. Further, the notation $\eta_{k} \stackrel{d}{\rightarrow} \eta$ means the weak convergence of the corresponding distributions with $\mathrm{P}\left\{\eta \in R^{n}\right\}=1$.

\subsection{Multiple Power Series Distributions}

First we give the necessary definitions. Let $\left(a(i) \geq 0, i \in Z_{+}^{n}\right)$ be a multiple sequence with

$$
B(x)=\sum_{i \in Z_{+}^{n}} a(i) x^{i}<\infty
$$

for $x=\left(x_{1}, \ldots, x_{n}\right) \in[0,1)^{n}$. The trivial case $a(i) \equiv 0, i \in Z_{+}^{n}$ is excluded. 
Definition 1. For $x \in[0,1)^{n}$ a random vector (r.v.) $\xi_{x}$ has a multiple power series distribution iff

$$
\mathrm{P}\left\{\xi_{x}=i\right\}=\frac{a(i) x^{i}}{B(x)}
$$

for some $B(x)$ and for any $i \in Z_{+}^{n}$.

It is clear that $\mathrm{P}\left\{\xi_{x} \in R^{n}\right\}=1$. The history of this notion and some bibliographic references are given in encyclopedias [2,3], also see the articles [20,22]. Let the sequence of vectors $b=b(k) \in$ $(0, \infty)^{n}, k \in N$ be given with $b_{j}=b_{j}(k) \rightarrow \infty, \forall j=1, \ldots, n$ as $k \rightarrow \infty$.

Definition 2 ([23]). We say that $B(x)$ regularly varies as $x \uparrow \mathbf{1}$ along the sequence $b=b(k)$, iff

$$
\frac{B(\exp (-\lambda / b))}{B(\exp (-\mathbf{1} / b))} \rightarrow \Psi(\lambda) \in(0, \infty)
$$

for an arbitrary fixed $\lambda=\left(\lambda_{1}, \ldots, \lambda_{n}\right)>0$ as $k \rightarrow \infty$.

(Notations $\lambda / b$ and $\exp (-\lambda / b)$ are defined in the Section 2.1).

The following statement has been proved in [21] (we formulate it as a lemma).

Lemma 1. A series $B(x)$ regularly varies as $x \uparrow \mathbf{1}$ along the sequence $b=b(k)$ iff for any (some) fixed vector $u \in G$ and $x=\exp (-u / b)$

$$
\xi_{x}(\mathbf{1}-x) \stackrel{d}{\rightarrow} \eta=\eta(u), \quad(k \rightarrow \infty)
$$

In both cases, the function $\Psi(\lambda)$ from Equation (2) is the Laplace transform of some $\sigma$-finite measure $\Phi(\cdot)$ and r.v. $\eta(u)$ has Laplace transform $\Psi((\lambda+\mathbf{1}) u) / \Psi(u)$.

Let $R(k)$ be some positive sequence. To formulate the resulting limit theorem, we need to give the following definition.

Definition 3. We say that the sequence $a(i)$ is $R$-weakly one-sided oscillatory at infinity along the sequence $b=b(k)$ if for every $j=1, \ldots, n$ and for any sequence $z_{j}=z_{j}(k)>1, z_{j}=1+o(1)$ one of the following inequalities

$$
\begin{gathered}
\liminf _{k \rightarrow \infty}\left(a\left(r_{1}, \ldots, r_{j-1}, z_{j} r_{j}, r_{j+1}, \ldots, r_{n}\right)-a(r)\right) / R(k) \geq 0 ; \\
\limsup _{k \rightarrow \infty}\left(a\left(r_{1}, \ldots, r_{j-1}, z_{j} r_{j}, r_{j+1}, \ldots, r_{n}\right)-a(r)\right) / R(k) \leq 0 .
\end{gathered}
$$

holds for every fixed $y=\left(y_{1}, \ldots, y_{n}\right) \in G$. Here $r=r(k)=\left(r_{1}(k), \ldots, r_{n}(k)\right)$ is an arbitrary function of $k$ with

$$
r_{1} \sim y_{1} b_{1}, \ldots, r_{n} \sim y_{n} b_{n}
$$

Hereinafter, we define $a(x)=a([x])$ for $x \notin Z_{+}^{n}$. The simplest examples of such sequences are monotone in each variable sequence $\left(a(i) \geq 0, i \in Z_{+}^{n}\right)$.

Theorem 1. Suppose that $B(x)$ regularly varies as $x \uparrow \mathbf{1}$ along the sequence $b=b(k)$ (i.e., the the assumption of integral limit Lemma 1 is true). Then, for any compact $K \subset G$ and for any (some) fixed vector $u \in G$ and $x=\exp (-u / b)$

$$
\frac{\mathrm{P}\left\{\xi_{x}=[y /(\mathbf{1}-x)]\right\}}{\prod_{j=1}^{n}\left(1-x_{j}\right)} \stackrel{y \in K}{\rightrightarrows} \psi_{u}(y)<\infty \quad(k \rightarrow \infty)
$$


where function $\psi_{u}(\cdot)$ is continuous in $G$, iff the sequence $a(i)$ is $R$-weakly one-sided oscillatory at infinity along the sequence $b=b(k)$ with

$$
R(k)=B(\exp (-\mathbf{1} / b(k))) / \prod_{i=1} b_{i}(k) .
$$

In both cases, the measure $\Phi(\cdot)$ from Lemma 1 has the continuous density $\varphi(\cdot)$ in $G$ and the following equality holds:

$$
\psi_{u}(y)=\frac{\varphi(y / u) e^{-(y, 1)}}{\prod_{j=1}^{n} u_{j} \Psi(u)}, \quad \forall y \in G
$$

Note that, in Theorem 1, the case when $\Phi(\partial G)>0(\Leftrightarrow \mathrm{P}\{\eta(u) \in \partial G\}>0)$ is not excluded. In addition, we admit that $\psi_{u}(y)=0, y \in V$, for some nonempty set $V \subseteq G$ in this theorem.

\section{Tauberian Lemma}

The next lemma gives some generalization of the Tauberian Theorem 2 from [23].

Lemma 2. Assume that $B(x)$ regularly varies as $x \uparrow 1$ along the sequence $b=b(k)$ (i.e., (2) holds). Then, for some continuous function $\varphi(\cdot)$ in $G$ the relation

$$
\frac{a(b v)}{R(k)} \stackrel{v \in K}{\rightrightarrows} \varphi(u)<\infty
$$

holds for any compact $K \subset G$ iff the sequence $a(i)$ is $R$-weakly one-sided oscillatory at infinity along the sequence $b=b(k)$ with $R(k)$ from Equation (7). In both cases, the measure $\Phi(\cdot)$ from Lemma 1 is absolutely continuous in $G$ with density $\varphi(\cdot)$.

Proof. For an arbitrary bounded set $A \subset R_{+}^{n}$, put

$$
\Phi_{k}(A)=\sum_{i \in Z_{+}^{n}, i / b \in A} \frac{a(i)}{\prod_{j=1}^{n} m_{j}(k) R(k)}
$$

It follows from Equations (2) and (7) that

$$
\begin{gathered}
\widetilde{\Phi}_{k}(y) \equiv \int_{R_{+}^{n}} e^{-(x, y)} \Phi_{k}(d x)=\frac{B(\exp (-y / b))}{\prod_{j=1}^{n} b_{j}(k) R(k)} \\
\rightarrow \psi(y)=\widetilde{\Phi}(y) \equiv \int_{R_{+}^{n}} e^{-(x, y)} \Phi(d x)
\end{gathered}
$$

for any fixed $y \in G$. The last equality follows from the statement of Lemma 1 . Thus, according to the continuity theorem for Laplace transforms of measures, it follows from Equation (10) that

$$
\Phi_{k}(\cdot) \Rightarrow \Phi(\cdot) .
$$

(see, for example the theorem 1.3.2 from [24]). Suppose that the sequence $a(i)$ is $R$-weakly one-sided oscillatory at infinity along the sequence $b=b(k)$. Set $m(j)=1$ if Equation (4) holds and $m(j)=-1$ if Equation (5) is valid. Fix $v \in G$. For an arbitrary $\delta \in(0,1)$, put

$$
A(\delta)=\left\{y=\left(y_{1}, \ldots, y_{n}\right), y_{j} \in\left(v_{j}, v_{j}(1+\delta)^{b(j)}\right), \forall j=1, \ldots, n\right\}
$$

(for $c>d$, we put $(c, d)=(d, c)$ ). Further, for an arbitrary $\varepsilon \in(0,1)$, there exists such $\delta \in(0, \varepsilon)$ that

$$
\frac{a(i)-a(b v)}{R(k)} \geq-\varepsilon
$$


for any $i \in m A(\delta)$. The proof of this fact repeats the proof of Lemma 5 from [23]. Without loss of generality, we assume that $\Phi(\partial A(\delta))=0$. It follows from Equations (9) and (13) that

$$
\begin{gathered}
\Phi_{k}(A(\delta))=\sum_{i \in Z_{+}^{n}, i / b \in A(\delta)} \frac{a(i)}{\prod_{j=1}^{n} b_{j}(k) R(k)} \\
\geq-\varepsilon+\frac{a(b v)}{R(k)} \frac{1}{\prod_{j=1}^{n} b_{j}(k)} \sum_{i \in Z_{+}^{n}, i / b \in A(\delta)} 1 . \\
\geq-\varepsilon+\frac{a(b v)}{R(k)}\left(1+\eta_{k}\right)|A(\delta)|
\end{gathered}
$$

where $\eta_{k} \rightarrow 0$ as $k \rightarrow \infty$. By $|A(\delta)|$, we denote here the Lebesque measure of the set $A(\delta)$. Therefore,

$$
\frac{a(b v)}{R(k)} \leq\left(\frac{\Phi_{k}(A(\delta))}{|A(\delta)|}+\frac{\varepsilon}{|A(\delta)|}\right) \frac{1}{1+\eta_{k}} .
$$

Since $\Phi(\partial A(\delta))=0$, we have from Equations (9) and (11) that

$$
\Phi_{k}(A(\delta))=\sum_{i \in Z_{+}^{n}, i / b \in A(\delta)} \frac{a(i)}{\prod_{j=1}^{n} b_{j}(k) R(k)} \rightarrow \Phi(A(\delta)) .
$$

Tending in Equation (14) $k$ to $\infty$ and using Equation (15), we have

$$
\limsup _{k \rightarrow \infty} \frac{a(b v)}{R(k)} \leq \frac{\Phi(A(\delta))}{|A(\delta)|}+\frac{\varepsilon}{|A(\delta)|}
$$

Since the left side of Equation (16) does not depend on $\varepsilon$, we have

$$
\limsup _{k \rightarrow \infty} \frac{a(b v)}{R(k)} \leq \frac{\Phi(A(\delta))}{|A(\delta)|} .
$$

Put $\Delta=\{\delta \in(0,1): \Phi(\partial A(\delta))=0\}$. Since the left side of Equation (17) does not depend on $\delta$, we have

$$
\limsup _{k \rightarrow \infty} \frac{a(b v)}{R(k)} \leq \liminf _{\delta \rightarrow 0, \delta \in \Delta} \frac{\Phi(A(\delta))}{|A(\delta)|} .
$$

Similarly, we obtain the inequality

$$
\liminf _{k \rightarrow \infty} \frac{a(b v)}{R(k)} \geq \limsup _{\delta \rightarrow 0, \delta \in \Delta} \frac{\Phi(A(\delta))}{|A(\delta)|} .
$$

It follows from Equations (18) and (19) that there exist the next two limits:

$$
\lim _{k \rightarrow \infty} \frac{a(b v)}{R(k)}=\lim _{\delta \rightarrow 0, \delta \in \Delta} \frac{\Phi(A(\delta))}{|A(\delta)|}(\stackrel{\text { def }}{=} \varphi(v)) .
$$

The next proof repeats the proof of Theorem 2 from [23]. The inverse assertion of Lemma 2 follows immediately from Equation (9). Lemma 2 is proved. 


\section{Proof of Theorem 1}

Suppose that Equation (2) holds. Put for $z \in N^{n}$ and $x \in[0,1]^{n}$

$$
p(z, x)=\mathrm{P}\left\{\xi_{x}=z\right\}=\frac{a(z)}{B(x)} \exp (z, \ln x) .
$$

We have

$$
a(z)=p(z, x) B(x) \exp (z,-\ln x) .
$$

Suppose that Equation (6) takes place for some $u \in G$ and continuous in $G$ function $\psi_{u}(\cdot)$. For fixed $y \in G$, put in Equation (20) $x=\exp (-u / b(k))$ and $z=[y /(\mathbf{1}-x)]$. We have $x=\mathbf{1}-(u+\varepsilon(k)) / b(k)$ and $z=[b(k) y /(u+\varepsilon(k))]=b(k)(y / u+\delta(k))$. Here $\varepsilon(k)$ and $\delta(k)$ are some functions tending to zero as $k \rightarrow \infty$. Thus $(z,-\ln x)=(y, \mathbf{1})+o(1)$ as $k \rightarrow \infty$. So, it follows from Equations (20), (2), and (6) that

$$
\begin{gathered}
a(z)=p(z, x) B(x) \exp (z,-\ln x)=(1+o(1)) p(z, x) B(x) \exp (y, \mathbf{1}) \\
=\prod_{j=1}^{n}\left(1-x_{j}\right) B(x)\left(\psi_{u}(y)+o(1)\right) \exp (y, \mathbf{1}) \\
=\prod_{j=1}^{n}\left(1-x_{j}\right) B\left(\exp (-\mathbf{1} / b(k)) \Psi(u)\left(\psi_{u}(y)+o(1)\right) \exp (y, \mathbf{1})\right. \\
=\prod_{j=1}^{n}\left(u_{j} / b_{j}(k)\right) B\left(\exp (-\mathbf{1} / b(k)) \Psi(u)\left(\psi_{u}(y)+o(1)\right) \exp (y, \mathbf{1})\right. \\
=R(k) \prod_{j=1}^{n} u_{j} \Psi(u)\left(\psi_{u}(y)+o(1)\right) \exp (y, \mathbf{1})
\end{gathered}
$$

according to Equation (7). Since Equation (6) holds locally uniformly on $y$ then it follows from Equation (21) that

$$
\frac{a(b y / u)}{R(k)} \rightarrow \prod_{j=1}^{n} u_{j} \Psi(u) \psi_{u}(y) \exp (y, \mathbf{1})=\varphi(y / u)
$$

and the last relation also holds locally uniformly on $y$. The equality Equation (8) follows directly from Equation (22). Replacing in Equation (22) $y / u$ by $v$, we obtain Equation (9). One-sided $R$-oscillation of $a(\cdot)$ along $b(k)$ follows immediately from Equation (9). The proof of inverse assertion repeats the proof of Theorem 2 from [20].

\section{On Some Previous Results}

The definition of regularly varying functions of one variable was given in Karamata's well-known work [25]. The notion of regularly varying functions at infinity along some sequence in an orthant was introduced in Omey [26]. The definition of regularly varying multiple power series is given in [23]. A brief overview of various definitions of multivariate regularly varying functions is available in [27]. The history of different class functions having slow (one-sided or ordinary) oscillation can be seen in the book [24]. In [22], we give the integral representation and Abelian statements (Theorems 3.1 and 3.2). With the help of these theorems, it is easy to set such sequences $a(i)$ explicitly.

As the source, for $n=1$ the sufficient condition for Equation (3) was given in Timashev [28], see also [8]. In [20], we show that conditions from [8,28] are equivalent to Equation (2). Timashev used the method of moments in his aforementioned result. In the papers [20,22,27] and in this article, we use the corresponding Tauberian statements. All these statements go back to Karamata's well-known Tauberian theorems [29,30]. 
Funding: This research received no external funding.

Acknowledgments: The author expresses his deep gratitude to reviewers for valuable comments.

Conflicts of Interest: The author declare no conflict of interest.

\section{References}

1. Noack, A. A class of random variables with discrete distributions. Ann. Math. Statist. 1950, 21, 127-132. [CrossRef]

2. Johnson, N.L.; Kotz, S.; Kemp, A.W. Univariate Discrete Distributions, 2nd ed.; Wiley Series in Probability and Statistics; John Wiley and Sons: New York, NY, USA, 1992.

3. Johnson, N.L.; Kotz, S.; Balakrishnan, N. Discrete Multivariate Distributions; Wiley Series in Probability and Statistics; Wiley: New York, NY, USA, 1997; Volume xxii, 299p.

4. Kolchin, V.F. A certain Class of Limit Theorems for Conditional Distributions. In Selected Translations in Mathematical Statistics and Probability; American Mathematical Society: Providence, RI, USA, 1973; Voume 11, pp. 185-197.

5. Kolchin, V.F. Random Mappings; Translations Series in Mathematics and Engineering; Optimization Software, Inc., Publications Division: New York, NY, USA, 1986.

6. Kolchin, V.F. Random Graphs, Encyclopedia Math. Appl. 53; Cambridge Univ. Press: Cambridge, UK, 1999.

7. Timashev, A.N. Random Components in Generalized Allocation Scheme; Akademiya: Moscow, Russia, 2017.

8. Timashev, A.N. Limit theorems for power-series distributions with finite radius of convergence. Theory Probab. Appl. 2018, 63, 45-56. [CrossRef]

9. Pavlov, Y.L. Random Forests; VSP: Utrecht, The Netherlands, 2000.

10. Chuprunov, A.N.; Fazekas, I. An analogue of the generalised allocation scheme: limit theorems for the number of cells containing a given number of particles. Discrete Math. Appl. 2012, 22, 101-122 [CrossRef]

11. Chuprunov, A.N.G.; Alsaied, M. Alkhuzani, On maximal quantity of particles of one color in analogs of multicolor urn schemes. Russ. Math. (Iz. VUZ) 2017, 61, 83-88. [CrossRef]

12. Pavlov, Y.L. Conditional configuration graphs with discrete power-law distribution of vertex degrees. Sb. Math. 2018, 209, 258-275. [CrossRef]

13. Pavlov, Y.L. On the connectedness of configuration graphs. Diskr. Mat. 2019, 31, 114-122.

14. Pavlov, Y.L. On the asymptotics of the cluster coefficient of a configuration graph with an unknown distribution of vertex degrees. Inform. Appl. 2019, 13, 9-13.

15. Pavlov, Y.L.; Cheplyukova, I.A. On the asymptotics of degree structure of configuration graphs with bounded number of edges. Discret. Math. Appl. 2019, 29, 219-232. [CrossRef]

16. Pavlov, Y.L.; Khvorostyanskaya, E.V. On the limit distributions of the degrees of vertices in configuration graphs with a bounded number of edges. Sb. Math. 2016, 207, 400-417. [CrossRef]

17. Leri, M.M.; Pavlov, Y.L. On the stability of configuration graphs in a random environment. Inform. Appl. 2018, 12, 2-10.

18. Bingham, N.H.; Goldie, C.M.; Teugels, J. Regular Variation; Cambridge University Press: Cambridge, UK, 1987.

19. Seneta, E. Regularly Varying Functions; Springer-Verlag: Berlin/Heidelberg, Germany; New York, NY, USA, 1976.

20. Yakymiv, A.L. On the multiple power series distribution regularly varying at the boundary point. Discret. Math. Appl. 2019, 29, 409-421. [CrossRef]

21. Yakymiv, A.L. Some Properties of Regularly Varying Functions and Series in the Orthant, Probability-Analytical models. In Methods and Applications, Springer Proceedings in Mathematics and Statistics; Shiryaev, A.N., Pavlov, I.V., Eds.; Springer: Berlin/Heidelberg, Germany, 2020. in press.

22. Yakymiv, A.L. Abelian theorem for the regularly varying measure and its density in orthant. Theory Probab. Appl. 2019, 64, 385-400. [CrossRef]

23. Yakymiv, A.L. A Tauberian theorem for multiple power series. Sb. Math. 2016, 207, 286-313. [CrossRef]

24. Yakymiv, A.L. Probabilistic Applications of Tauberian Theorems.-Modern Probability and Statistics; VSP: Leiden, The Netherlands; Boston, MA, USA, 2005; Volume viii, 225p.

25. Karamata, J. Sur un mode croissanse régulière des fonctions. Mathematica 1930, 4, 38-53. 
26. Omey, E. Multivariate Regular Variation and Application in Probability Theory, Eclectica; EHSAL: Brussel, Belgium, 1989; Volume 74.

27. Yakymiv A.L. Multivariate Regular Variation in Probability Theory. J. Math. Sci. 2020, 246, 580-586. [CrossRef]

28. Timashev, A.N. Power Series Distributions and Generalized Allocation Scheme; Akademiya: Moscow, Russia, 2016.

29. Karamata, J. Über die Hardy-Littelwoodsche Umkehrungen des Abelschen Steitigkeitssatzes. Math. Z. 1930, 32, 319-320. [CrossRef]

30. Karamata, J. Neuer Beweis und Verallgemeinerung einiger Tauberian-Sätze. Math. Z. 1931, 33, $294-299$. [CrossRef]

Publisher's Note: MDPI stays neutral with regard to jurisdictional claims in published maps and institutional affiliations.

(C) 2020 by the authors. Licensee MDPI, Basel, Switzerland. This article is an open access article distributed under the terms and conditions of the Creative Commons Attribution (CC BY) license (http:/ / creativecommons.org/licenses/by/4.0/). 